sind wir für die Überlassung und Reinigung von Substanzen sehr verpflichtet. Dem Direktor des Instituts für Angewandte Mathematik, Freiburg, Herrn Prof. GörtLER, sowie Herrn Dr. GEIS danken wir für die Erlaubnis und Anleitung zur Benutzung des Rechenautomaten Z22. A n m. b. d. Ko r r. :

1. Während der Drucklegung wurde ein ähnliches Analysierverfahren für $\mathrm{A}_{2} \mathrm{~B}_{2}$-Spektren veröffentlicht von B. D. Nageswara Rao u. P. Venkateswarlu, Proc. Ind. Acad. Sci. 54 A, 1 [1961]. - Auch dort werden die Parameter allein aus den Linienlagen berechnet, d. h. ohne Anpassung eines berechneten an das gemessene Spektrum.

2. In den beiden $\mathrm{A}_{2} \mathrm{~B}_{2} \mathrm{X}$-Spektren ( $p$-Fluornitrobenzol und Pyrrol) konnten wir neuerdings unter Sättigungsbedingungen auch Mehrquantenübergänge beobachten (vgl. ${ }^{6}$, S. 31). Wie an anderer Stelle noch gezeigt werden soll, läßt sich daraus die Größe von

$\left|J_{\mathrm{AX}}+J_{\mathrm{BX}}\right|$ bestimmen. Die Ergebnisse bestätigen unsere Annahme, daß bei den beiden Substanzen die Kopplungskonstanten $J_{\mathrm{AX}}$ und $J_{\mathrm{BX}}$ jeweils gleiche relative Vorzeichen besitzen.

\title{
Elektron-Spin-Resonanz des Dibenzolvanadins
}

\author{
Von K. H. Hausser \\ Aus dem Max-Planck-Institut für Medizinische Forschung, Institut für Chemie, Heidelberg \\ (Z. Naturforschg. 16 a, 1190-1192 [1961] ; eingegangen am 12. August 1961)
}

\begin{abstract}
Beim Dibenzolvanadin gelingt die Auflösung der Hyperfeinstruktur infolge Kopplung mit dem Zentralatom und mit den Protonen der Benzolringe. Die für den $g$-Faktor und die HyerfeinstrukturKopplungskonstanten gemessenen Werte stimmen gut mit einer Theorie 2. Ordnung überein. Die Ergebnisse werden mit den an anderen Sandwich-Komplexen erhaltenen verglichen.
\end{abstract}

Bei einer Reihe von paramagnetischen SandwichKomplexen wurde eine Hyperfeinstruktur (HFS) der Elektron-Spin-Resonanz (ESR) beobachtet ${ }^{1}$, die von der Kopplung des ungepaarten Elektrons mit den Ringprotonen herrührt. Beim Dicyclopentadienyl-Vanadin erhielt McConnell ${ }^{2}$ infolge Kopplung mit dem $\mathrm{V}^{51}$-Kern (Spin $\left.I=7 / 2\right) 8$ äquidistante HFS-Komponenten gleicher Intensität und Linienbreite im Abstand von 27,4 Gauß. Eine Auflösung der durch das Zentralatom und durch die Ringprotonen verursachten HFS in derselben Verbindung ist bisher nur beim Dibenzolchromium-Jodid gelungen ${ }^{3}$. Bei der ESR dieser Verbindung treten vor und nach der Hauptgruppe zwei identische Satellitengruppen auf, die dem seltenen Chrom-Isotop $\mathrm{Cr}^{53}$ (natürliche Häufigkeit $9,5 \%$, Spin $I=3 / 2$ ) zugeordnet werden konnten. In der vorliegenden Arbeit konnte sowohl die HFS auf Grund der Kopplung mit dem $\mathrm{V}^{51}$-Kern als auch die durch die 12 äquivalenten Protonen der Benzolringe verursachte HFS aufgelöst werden.

\section{Experimentelles}

Die Untersuchungen wurden an einer sauerstofffreien Lösung von Dibenzolvanadin in einem Gemisch von Toluol und Methylcyclohexan (1:1), Konzentration

1 a) R. D. Feltham, P. Sogo u. M. Calvin, J. Chem. Phys. 26, 1354 [1957] ; b) B. Elschner, Z. Naturforschg. 12 a, 860 [1957] ; c) K. H. Hausser, Naturwiss. 48, 426 [1961]. ca. $10^{-3} \mathrm{Mol} / l$, bei Temperaturen zwischen $+40{ }^{\circ} \mathrm{C}$ und $-40^{\circ} \mathrm{C}$ durchgeführt. Für die Überlassung der Meßsubstanz bin ich Herrn Prof. E. O. Fischer zu großem Dank verpflichtet. Die Mikrowellenfrequenz des zur Messung verwendeten Varian-ESR-Spektrometers betrug ca. $9450 \mathrm{MHz}$, die Frequenz der Feldmodulation $100 \mathrm{kHz}$. Zur Bestimmung des g-Faktors und des Abstandes zwischen den einzelnen Liniengruppen wurde die Mikrowellenfrequenz durch Überlagerung mit einer Oberwelle einer quarzgesteuerten Frequenzdekade (Schomandl FD 3) und das Magnetfeld durch Messung der Protonen-Resonanzfrequenz ebenfalls mit einer quarzgesteuerten Frequenzdekade (Rohde u. Schwarz XUD) gemessen. Die Genauigkeit von etwa 1 Teil in $10^{4}$ des $g$-Faktors wird dadurch begrenzt, wie genau sich das Magnetfeld auf die Mitte der ESR-Linie einstellen ließ.

\section{Ergebnisse und Diskussion}

Das ESR-Spektrum des Dibenzol-Vanadins ist in Abb. 1 bei $+20^{\circ} \mathrm{C}$ und bei $-40{ }^{\circ} \mathrm{C}$ dargestellt. Das Spektrum besteht aus 8 Liniengruppen entsprechend dem Spin $I=7 / 2$ des Zentralatoms; die einzelnen Gruppen zeigen eine zusätzliche, nur teilweise aufgelöste Struktur infolge Kopplung mit den Protonen der Benzolringe. Dabei fällt besonders auf, daß die einzelnen den verschiedenen magnetischen Quantenzahlen $m_{I}$ des Zentralatoms zuzuordnenden

2 H. W. McConnell, W. W. Porterfield u. R. E. Robertson, J. Chem. Phys. 30, 442 [1959].

${ }^{3}$ K. H. Hausser, Naturwiss. 48, 666 [1961]. 

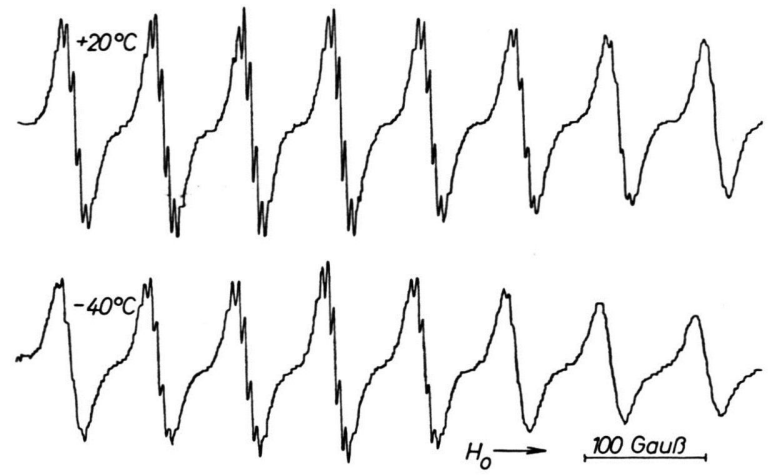

Abb. 1. ESR-Spektrum des Dibenzolvanadins in Toluol : Methylcyclohexan $(1: 1)$, Konzentration $c=10^{-3} \mathrm{Mol} / l$, bei $+20^{\circ} \mathrm{C}$ und bei $-40{ }^{\circ} \mathrm{C}$.

HFS-Komponenten eine verschiedene Linienbreite und dementsprechend verschiedene Auflösung der HFS haben; dieser Effekt wird bei tieferer Temperatur, höherer Viskosität noch ausgeprägter. Eine ähnliche Abhängigkeit der Linienbreite von der magnetischen Quantenzahl $m_{I}$ fanden auch PAKE und Mitarbeiter beim $\mathrm{VO}^{++}$-Ion ${ }^{4}$ und Powles beim Disulfonat-Ion $\left(\mathrm{SO}_{3}\right)_{2} \mathrm{NO}^{--5}$.

Die Ursache liegt nach einem Vorschlag von McConnell $^{6}{ }^{6}$, der von Kivelson ${ }^{7}$ weiter ausgeführt wurde, in der Anisotropie des $g$-Faktors und der HFS-Wechselwirkung. Dazu muß man eine NahOrientierung der Wassermoleküle in der Nähe des Ions annehmen, so daß dieses nicht mehr als freies Ion, sondern in einem „Mikrokristall“ vorliegt.

In dem hier vorliegenden Fall, in dem sich das Vanadin-Atom im Zentrum eines kovalent gebundenen Moleküls befindet, ist eine solche Anisotropie des $g$-Faktors und der HFS-Wechselwirkung sicher ohne besondere Annahmen gegeben; bei dem ähnlich gebauten Dibenzol-chromium-Ion ergab sich für die Anisotropie des $g$-Faktors $g_{\|}-g_{\perp}=0,016 \pm 0,001^{1 \mathrm{c}}$ : Es fällt auf, daß beim $\mathrm{V}^{++}$-Ion, wo keine zusätzliche Aufspaltung der 8 Linien beobachtet wird, die kleinste gemessene Linienbreite etwa $12 \mathrm{Gau} \beta$ beträgt, während die Linienbreite der einzelnen HFS-Komponenten in Abb. 1 wesentlich kleiner ist.

Den zeitunabhängigen Hamilton-Operator kann man bei Vernachlässigung der Wechselwirkung mit den Ringprotonen in der Form schreiben

$$
\mathcal{H}=\langle g\rangle \mu_{\mathrm{B}} \vec{H}_{0} \cdot \vec{S}+\langle a\rangle \vec{I} \cdot \vec{S} .
$$

${ }^{4}$ R. N. Rogers u. G. E. Pake, J. Chem. Phys. 33, 1107 [1960]. 5 J. G. Powles u. M. H. Mosley, Proc. Phys Soc., Lond. A 77, 729 [1961].
Bei Beschränkung auf axiale Symmetrie, die bei den Sandwich-Komplexen in guter Näherung gegeben ist, beträgt der gemittelte $g$-Faktor

$$
\langle g\rangle=(1 / 3)\left(g_{\|}+2 g_{\perp}\right)
$$

und die gemittelte HFS-Kopplungskonstante

$$
\langle a\rangle=(1 / 3)\left(a_{\|}+2 a_{\perp}\right) .
$$

Die Eigenwerte in zweiter Ordnung sind ${ }^{4}$

$$
\begin{aligned}
h v=\langle g\rangle \mu_{\mathrm{B}} H_{0}+\langle a\rangle & m_{I} \\
& +\frac{\langle a\rangle^{2}}{2\langle g\rangle \mu_{\mathrm{B}} H_{0}}\left[I(I+1)-m_{I}^{2}\right] .
\end{aligned}
$$

Der scheinbare $g$-Faktor, bezogen auf den Mittelpunkt zwzischen den beiden mittleren Linien, ergibt sich zu 1,9920; unter Berücksichtigung der Verschiebung durch den Term zweiter Ordnung in (2) erhält man für den $g$-Faktor :

$$
\langle g\rangle=1,9866 \pm 0,0002 \text {. }
$$

Der $g$-Faktor ist merklich verschieden von demjenigen des freien Ions $(1,9624){ }^{4}$. Er stimmt jedoch ausgezeichnet mit dem des Dibenzol-chromiumIons $(1,9863)$ überein ${ }^{1 \mathrm{c}}$ entsprechend der isoelektronischen Struktur dieser beiden Verbindungen.

Der Korrektur-Term 2. Ordnung in (2) verschwindet für die Differenz der beiden den kernmagnetischen Quantenzahlen $m_{I}= \pm 1 / 2$ entsprechenden Energieniveaus. Der Abstand zwischen der 4. und 5. HFS-Liniengruppe ergibt also direkt die HFS-Kopplungskonstante zwischen dem ungepaarten Elektron und dem $\mathrm{V}^{51}$-Kern; man erhält für $\langle a\rangle$, ausgedrückt in Gauß:

$$
\langle a\rangle=63,5 \pm 1,0 \mathrm{Gau} 3 .
$$

Mit (4) und mit $H_{0}=3400 \mathrm{Gauß}$ wird der Korrektur-Term 2. Ordnung für den Abstand zwischen zwei HFS-Niveaus mit den kernmagnetischen Quantenzahlen $m_{I}$ und $m_{I}^{\prime}\left(m_{I}>m_{I}^{\prime}\right)$ :

$$
\Delta\langle a\rangle=0,6\left(m_{l}^{\prime 2}-m_{l}^{2}\right) \text { Gauß. }
$$

Innerhalb der Meßgenauigkeit stimmt dieser Wert mit dem gemessenen ESR-Spektrum in Abb. 1 sehr gut überein.

Die Kopplungskonstante $\langle a\rangle$ ist sehr verschieden von derjenigen des freien Ions und ebenfalls von der im Dicyclopenta-dienyl-vanadin gemessenen. Wegen der Bedeutung für die Bindungstheorie der

6 H. M. McConnell, J. Chem. Phys. 25, 709 [1956].

7 D. Kivelson, J. Chem. Phys. 33, 1094 [1960]. 
Sandwich-Komplexe sind die gemessenen Kopplungskonstanten einiger Vanadinverbindungen in Tab. 1 zusammengestellt:

\begin{tabular}{|l|c|c|}
\hline \multicolumn{1}{|c|}{$\begin{array}{c}\text { Vanadin- } \\
\text { Verbindung }\end{array}$} & $\begin{array}{c}\text { Kopplungskonstante } \\
\langle a\rangle(\mathrm{Gau})\end{array}$ & Literatur \\
\hline $\mathrm{VO}^{++}$-Ion & 118 & 4 \\
Oxydationsprodukt & 75 & 2 \\
von V $\left(\mathrm{C}_{5} \mathrm{H}_{5}\right)_{2}$ & 63 & \\
$\mathrm{~V}\left(\mathrm{C}_{6} \mathrm{H}_{6}\right)_{2}$ & 60 & 8 \\
$\mathrm{~K}_{4} \mathrm{~V}\left(\mathrm{CNN}_{6}\right)_{6}$ & 27 & 2 \\
$\mathrm{~V}\left(\mathrm{C}_{5} \mathrm{H}_{5}\right)_{2}$ & & \\
\hline
\end{tabular}

Tab. 1.

Die Aufenthaltswahrscheinlichkeit des ungepaarten Elektrons am Vanadin-Kern ist demnach beim Hexacyanokomplex dem Dibenzol-vanadin am ähnlichsten, während sie beim Dicyclopenta-dienyl-vanadin wesentlich kleiner ist. Sie sollte daher bei letzterem außen in den Ringen entsprechend größer sein und zu einer auflösbaren Protonen-Hyperfeinstruktur führen.

Für die HFS-Kopplungskonstante $\langle a\rangle_{\mathrm{P}}$ zwischen dem ungepaarten Elektron und den Ringprotonen

8 J. M. Baker u. B. Bleaney, Proc. Phys. Soc., Lond. A 65, 952 [1952]. des Dibenzol-vanadins ergibt sich $\langle a\rangle_{\mathrm{P}}=4,0 \pm 0,1$ Gauß. Der entsprechende Wert für Dibenzol-chromium-jodid ist $3,25 \mathrm{Gau}{ }^{1 \mathrm{c}}$; daraus erhält man für das Verhältnis $\langle a\rangle_{\mathrm{P}(\mathrm{Cr})} /\langle a\rangle_{\mathrm{P}(\mathrm{V})}=0,81$. Umgekehrt ist bei der Chrom-Verbindung die Kopplung mit dem Zentralatom enger, das Verhältnis der Kopplungskonstanten beträgt bei Berücksichtigung der verschiedenen magnetischen Momente

$$
\left(\frac{\langle a\rangle \cdot I}{\mu_{I}}\right)_{\mathrm{v}}:\left(\frac{\langle a\rangle \cdot I}{\mu_{I}}\right)_{\mathrm{Cr}}=0,80
$$

es stimmt gut mit dem umgekehrten Verhältnis bei den Protonen überein. Das ungepaarte Elektron befindet sich also beim Dibenzol-chromium-Ion mehr in der Nähe des Zentralatoms, während es beim Dibenzol-vanadin eine etwas größere Aufenthaltswahrscheinlichkeit in den beiden Benzolringen besitzt. Dieses Ergebnis ist verständlich, wenn man bedenkt, $\mathrm{da} ß$ es sich um zwei isoelektronische Verbindungen handelt, die sich nur durch die beim Dibenzol-chromium-Ion um eine Einheit höhere positive Kernladung unterscheiden.

Herrn Dr. J. Powles danke ich für eine Diskussion der Ergebnisse, Herrn A. Überle für seine Hilfe bei der Durchführung der Messungen, der Deutsehen Forschungsgemeinschaft für die finanzielle Unterstützung der Arbeit.

\title{
Fluoreszenzabklingdauer von einigen aromatischen Kohlenwasserstoffen bei $\alpha$-Strahlanregung
}

\author{
Von A. Schmillen und K. Kramer \\ Aus dem Physikalischen Institut der Universität Gießen \\ (Z. Naturforschg. 16 a, 1192-1199 [1961] ; eingegangen am 5. August 1961)
}

\begin{abstract}
Aus dem Fourier-Spektrum von Szintillationslichtblitzen in a-bestrahlten kristallinen Pulvern aromatischer Kohlenwasserstoffe werden Aussagen über die Abklingzeiten (zum Teil auch über Anklingzeiten) gewonnen. Die reinen Kristallpulver zeigen alle ein nicht einfach exponentielles Abklingen der Lumineszenzblitze. Dagegen findet man für die Lumineszenz der Fremdmoleküle, die in die gleichen Wirtsgitter eingebaut wurden, ein fast einfach exponentielles Abklingen. Die gemessenen Werte für die Abklingzeiten sind in zwei Tabellen zusammengestellt. Konsequenzen, die sich daraus für den Anregungsmechanismus ergeben, werden kurz diskutiert.
\end{abstract}

Nicht nur unter dem Gesichtspunkt der Anwendung in der Szintillometrie, sondern auch für die Aufklärung der physikalischen Vorgänge bei der Lumineszenzanregung ist die Untersuchung organischer Leuchtstoffe auf ihr Abklingverhalten von großem Interesse. Wir berichten im folgenden über das Abklingen von Szintillationsblitzen, die von $\alpha$-Teilchen in fluoreszierenden festen Kohlenwasserstoffen ausgelöst werden. Der Intensitätsverlauf der Licht- blitze wurde durch Aufnahme des Fourier-Spektrums festgestellt, ein Verfahren, das seinerzeit von $\mathrm{GLÄSER}^{1}$ angegeben und versucht wurde. Zwar gibt das Fourier-Spektrum keine volle, eindeutige Information über den zeitlichen Intensitätsverlauf, es läßt aber erkennen, ob derselbe einem einfachen exponentiellen Abfall folgt oder nicht; in letzterem Falle

1 F. GLÄSER, Z. Naturforschg. 11 a, 1030 [1956]. 